\title{
A importância da qualidade do sono no processo de aprendizagem em estudantes da área de saúde: uma revisão integrativa
}

\author{
The importance of sleep quality in the learning process of medical students: a integrative review
}

La importancia de la calidad del sueño en el proceso de aprendizaje de los estudiantes de medicina: una revisión integradora

Recebido: 23/08/2021 | Revisado: 29/08/2021 | Aceito: 30/08/2021 | Publicado: 03/09/2021

Sophia Kelly Abreu de Castro

ORCID: https://orcid.org/0000-0003-3316-0046

Universidade Federal de Alfenas, Brasil

E-mail: sophia.castro@sou.unifal-mg.edu.br

Stela Lima Mulati

ORCID: https://orcid.org/0000-0003-1735-3040 Universidade Federal de Alfenas, Brasil

E-mail: stela.mulati@sou.unifal-mg.edu.br

Gema Galgani de Mesquita Duarte

ORCID: https://orcid.org/0000-0002-4899-5479 Universidade Federal de Alfenas, Brasil E-mail: gema.duarte@unifal-mg.edu.br

Tales Alexandre Aversi-Ferreira

ORCID: https://orcid.org/0000-0002-4417-7209 Universidade Federal de Alfenas, Brasil E-mail: aversiferreira@gmail.com

\begin{abstract}
Resumo
O sono é primordial para o funcionamento do organismo e suas funções vitais, repercutindo de maneira complexa no cotidiano dos indivíduos. A privação do sono e alterações de sua qualidade afetam negativamente diversos âmbitos. Estudantes de medicina são uma classe normalmente acometida por tal privação, visto a carga horária elevada e o estilo de vida fatigante, exibindo consequências na vida acadêmica, profissional e pessoal. Este artigo exibe os impactos da privação do sono frente às adversidades do curso, com consequências na aprendizagem, rendimento e cognição dos estudantes. A revisão integrativa foi desenvolvida através dos métodos em etapas de Identificação dos objetivos; planejamento do protocolo; estratégias de busca nas plataformas MEDLINE, Lilacs, SciELO e Google Acadêmico; incluídos trabalhos com temática na alteração do sono em estudantes de medicina, seu impacto na aprendizagem e/ou empecilhos diretamente relacionados à privação, de até 10 anos de publicação. As mudanças para alcance de uma formação médica de qualidade, enfocada na saúde psíquica e na qualidade de vida do estudante, envolvem tanto a comunidade médica, a esfera cultural e principalmente as instituições de ensino.
\end{abstract}

Palavras-chave: Privação do sono; Neurofisiologia; Neuropsicologia; Sono; Estudantes da saúde.

\begin{abstract}
Sleep is essential for the functioning of the organism and its vital functions, having a complex impact on the daily lives of individuals. Sleep deprivation and changes in its quality negatively affect several areas. Medical students are a class normally affected by such deprivation, given the high workload and strenuous lifestyle, showing consequences in academic, professional and personal life. This article shows the impacts of sleep deprivation in the face of course adversities, with consequences on students' learning, performance and cognition. The integrative review was developed through the methods in stages of Identification of objectives; protocol planning; search strategies on MEDLINE, Lilacs, SciELO and Academic Google platforms; Works on sleep disorders in medical students, its impact on learning and/or obstacles directly related to deprivation, up to 10 years of publication, were included. Changes to achieve quality medical training, focused on mental health and quality of life for students, involve both the medical community, the cultural sphere and, above all, educational institutions.
\end{abstract}

Keywords: Sleep deprivation; Neurophysiology; Neuropsychology; Sleep; Health’s students.

\section{Resumen}

El sueño es fundamental para el funcionamiento del organismo y sus funciones vitales, teniendo un impacto complejo en la vida diaria de las personas. La falta de sueño y los cambios en su calidad afectan negativamente a varias áreas. Los estudiantes de medicina son una clase que normalmente se ve afectada por tal privación, dada la alta carga de trabajo y el estilo de vida extenuante, que muestra consecuencias en la vida académica, profesional y personal. Este artículo 
muestra los impactos de la privación del sueño frente a las adversidades del curso, con consecuencias en el aprendizaje, el rendimiento y la cognición de los estudiantes. La revisión integradora se desarrolló a través de los métodos en etapas de Identificación de objetivos; planificación de protocolos; estrategias de búsqueda en las plataformas MEDLINE, Lilacs, SciELO y Academic Google; Se incluyeron trabajos sobre trastornos del sueño en estudiantes de medicina, su impacto en el aprendizaje y / u obstáculos directamente relacionados con la privación, hasta los 10 años de publicación. Los cambios para lograr una formación médica de calidad, centrada en la salud mental y la calidad de vida de los estudiantes, involucran tanto a la comunidad médica, al ámbito cultural y, sobre todo, a las instituciones educativas.

Palabras clave: Privación del sueño; Neurofisiología; Neuropsicología; Sueño, Estudiantes de la Salud.

\section{Introdução}

O sono se refere a um estado fisiológico essencial aos organismos, principalmente para os mamíferos, e se refere a um comportamento altamente complexo com vários problemas a serem resolvidos pelas neurociências (Aloé, Azevedo \& Hasan, 2005); e é divido em ciclos ou estágios atribuídos ao sistema hipotalâmico que envolve o ciclo circadiano (Mignot, Taheri \& Nishino, 2002; Pace-Schott \& Hobson, 2002).

As classificações desses estágios foram identificadas pelos movimentos oculares e classificados em REM [do inglês Rapid Eyes Movement] e NREM [N significa no] em função de estudos de eletroencefalograma [EEG] (Aloé, Azevedo \& Hasan, 2005). O estágio NREM é dividido em quatro fases de acordo com a profundidade do sono, com 3 estágios e mais um mostrando um sono de ondas lentas ou sono delta. O estágio REM mostra ondas baixas e dessincronizadas (Peever, 2017).

O comportamento complexo do sono envolve fenômenos fisiológicos e sociais importantes como uma forma efetiva de consolidação de memórias e ideias, fornecendo estabilização e sua integração no processo de armazenamento de memórias a longo prazo (Ackermann, 2014).

As características observadas no EEG são importantes para entender os problemas do sono em seres humanos, pois a manutenção dos estágios citados é primordial para a qualidade do sono e, logo, para uma melhor qualidade de vida.

Como comparação, em modelos murinos submetidos a $5 \mathrm{~h}$ de privatização por dia em várias semanas do foi observado déficit da aprendizagem reversa, das tarefas motoras, de memória e aprendizado (Varga, 2014), em humanos, estados de ansiedade, déficit de atenção e agressividade (Pires, Tufik \& Andersen, 2012), alterações de humor de processos emocionais dificultando a tomada de decisões, aumenta a incidência de erros e risco de acidentes, aumenta a sonolência, pode causar problemas como o aumento de peso, diabetes tipo 2, hipertensão (Landolt, Sousek \& Holst, 2015).

Em termos neuropsicológicos, o controle do sono é realizado pela chamada I Unidade Funcional do Cérebro de Luria (Sugahara et al., 2021; Cordeiro-de-Oliveira, 2021; Aversi-Ferreira et al., 2010; Aversi-Ferreira et al., 2019; Luria, 1973) que esta localizada principalmente no tronco encefálico com ricas conexões com a parte mesial do lobo frontal (Luria, 1973) e que estão ligados via circuito tálamo-cortical (Aloé, Azevedo \& Hasan, 2005). Essas relações indicam de modo geral as áreas cerebrais que podem ser afetadas pela privação do sono.

Dentro desse escopo geral e especificando para condições de profissionais que precisam controlar o sono em suas profissões como os da área da saúde formam uma parcela específica mais suscetível à privação de sono devido às elevadas cargas horárias laborais desempenhadas e, dentro dessa análise, os estudantes dessas áreas também devem ser considerados (Ribeiro, 2014), pois a alteração do ritmo circadiano está acontecendo inicialmente na universidade.

Portanto, o objetivo dessa revisão foi discutir sobre a privação do sono nos estudantes da área de saúde.

\section{Metodologia}

Foi realizada uma revisão integrativa da literatura especializada para reunir e compilar e discutir sobre o conhecimento científico da qualidade do sono em estudantes da área de saúde. 
Foram realizadas biscas de artigos nas bases de dados dos periódicos Capesn que envolveram as bases PubMed, Scopus, Elsevier, WebScience, SciELO e, também pela base Google Acadêmico no período de junho a agosto de 2021 (CAPES). A terminologia em saúde foi derivada dos Descritores em Ciências da Saúde (DECS) e no Medical Subject Headings (MeSH), nos idiomas português, inglês e espanhol. Os temas utilizados nas buscas foram: privação do sono, neurofisiologia e ou neuropsicologia, sono e estudantes da área da saúde.

Os critérios de inclusão adotados pelo presente estudo foram: publicação de temática que relacionasse o sono e à sua privação; publicações classificadas como artigo original divulgadas em língua inglesa, espanhola e portuguesa; livro clássico de neuropsicologia e 1 capítulo de livro, totalizando 22 textos. Foram excluídos os editoriais, as cartas ao editor e estudos que não abordaram a temática da revisão. Os textos de neuropsicologia e neurofisiologia foram usados para fornecer a sustentação epistemológica ao trabalho.

Foi feita a elaboração cuidadosa da síntese a partir das evidências disponíveis nas obras, mostrando as diversas consequências da privação do sono e seus impactos relacionados à aprendizagem dos graduandos dos cursos de saúde.

\section{Resultados e Discussão}

Após uma pesquisa cuidadosa foram selecionadas as obras para a leitura na íntegra, permanecendo um total de 22 artigos que preencheram os critérios de inclusão e foram efetivamente selecionados e revisados para compor a amostra.

A Tabela 1 representa a síntese das informações relacionadas aos 22 estudos utilizados nesse trabalho.

Tabela 1: Apresentação da síntese dos artigos incluídos na Revisão Sistemática: Identificação do estudo, autores, fontes de informação, periódicos e ano. Alfenas/MG, 2021.

\begin{tabular}{|c|c|c|c|}
\hline & Textos & Temas & Tipos de estudos \\
\hline 1. & $\begin{array}{l}\text { Ackermann, S. \& Rasch, B. (2014). Differential Effects of Non- } \\
\text { REM and REM Sleep on Memory Consolidation? Current } \\
\text { Neurology and Neuroscience Reports, 14(2). }\end{array}$ & Privação do sono & Revisão \\
\hline 2. & $\begin{array}{l}\text { Ahrberg, K., Dresler, M., Niedermaier, S., Steiger, A., \& Genzel, L. } \\
(2012) \text {. The interaction between sleep quality and academic } \\
\text { performance. Journal of Psychiatric Research, 46(12), 1618-1622. }\end{array}$ & Privação do sono & $\begin{array}{l}\text { Quantitativo e } \\
\text { qualitativo }\end{array}$ \\
\hline 3. & $\begin{array}{l}\text { Al-Khani, A. M., Sarhandi, M. I., Zaghloul, M. S., Ewid, M., \& } \\
\text { Saquib, N. (2019). A cross-sectional survey on sleep quality, mental } \\
\text { health, and academic performance among medical students in Saudi } \\
\text { Arabia. BMC Research Notes, 12(1). }\end{array}$ & Privação do sono & Transversal \\
\hline 4. & $\begin{array}{l}\text { Aloé, F., Azevedo, A. P. \& Hasan, R. (2005). Sleep-wake cycle } \\
\text { mechanisms. Revista Brasileira de Psiquiatria, 27(supl. I), 33-39. }\end{array}$ & $\begin{array}{l}\text { Neurofisiologia/neuropsicolog } \\
\text { ia }\end{array}$ & Revisão \\
\hline 5. & $\begin{array}{l}\text { Alvarado Fernández, V. et al., (2012). Impact of sleep deprivation } \\
\text { on the cognitive abilities of a population of medical students. Med. } \\
\text { Leg. Costa Rica ; } 29(2): 19-38\end{array}$ & $\begin{array}{l}\text { Privação do sono, } \\
\text { neurofisiologia/neuropsicologi } \\
\text { a }\end{array}$ & $\begin{array}{l}\text { Transversal } \\
\text { qualitativo }\end{array}$ \\
\hline 6. & $\begin{array}{l}\text { Aversi-Ferreira, T. A., Araújo, M. F. P. D., Lopes, D. B., \& } \\
\text { Nishijo, H. (2010). History, citoarchitecture and neurophysiology } \\
\text { of human and non human primates' parietal lobe: A review. } \\
\text { Dementia \& neuropsychologia, 4(3), 173-180. }\end{array}$ & $\begin{array}{l}\text { Neurofisiologia/neuropsicolog } \\
\text { ia }\end{array}$ & Revisão \\
\hline 7. & $\begin{array}{l}\text { Aversi-Ferreira, T. A., Tamaishi-Watanabe, B. H., Magri, M. P. D. } \\
\text { F., \& Aversi-Ferreira, R. A. (2019). Neuropsychology of the } \\
\text { temporal lobe: Luria's and contemporary conceptions. Dementia \& } \\
\text { neuropsychologia, 13(3), 251-258. }\end{array}$ & $\begin{array}{l}\text { Neurofisiologia/neuropsicolog } \\
\text { ia }\end{array}$ & Revisão \\
\hline
\end{tabular}




\begin{tabular}{|c|c|c|c|}
\hline 8. & $\begin{array}{l}\text { Castilho, C. P., Limas, L. M. D. de, Monteiro, M. L., Silva, P. H. } \\
\text { M. N. da, Bueno, H., \& Fari, T. A. (2015). A privação de sono nos } \\
\text { alunos da área de saúde em atendimento nas Unidades Básicas de } \\
\text { Saúde e suas consequências. Revista De Medicina, 94(2), 113-119. }\end{array}$ & $\begin{array}{l}\text { Privação do sono, sono e } \\
\text { estudantes da área da saúde }\end{array}$ & Transversal \\
\hline 9. & $\begin{array}{l}\text { Coelho, A. P. S. et al., (2020). Saúde mental e qualidade do sono de } \\
\text { universitários na época da pandemia do COVID-19: experiência de } \\
\text { um programa de assistência estudantil. Research, Society and } \\
\text { Development, } 9 \text { (9), e943998074. }\end{array}$ & $\begin{array}{l}\text { Privação do sono, sono e } \\
\text { estudantes da área da saúde }\end{array}$ & $\begin{array}{l}\text { Descritivo } \\
\text { Qualitativo }\end{array}$ \\
\hline 10. & $\begin{array}{l}\text { Cordeiro-de-Oliveira, K. ., Souza-Couto, D., Caixeta, M., Caixeta, } \\
\text { V., \& Aversi-Ferreira, T. A. (2021). Neuropsychology of the frontal } \\
\text { lobe and III functional brain unit: A Luria's studies and perspectives } \\
\text { for the clinic approach. Research, Society and Development, 10(7), } \\
\text { e48210716760. https://doi.org/10.33448/rsd-v10i7.16760 }\end{array}$ & 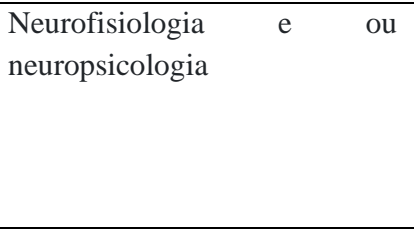 & Revisão \\
\hline 11. & $\begin{array}{l}\text { Corrêa, Camila de Castro et al. Sleep quality in medical students: a } \\
\text { comparison across the various phases of the medical course. Jornal } \\
\text { Brasileiro de Pneumologia [online]. 2017, v. } 43 \text {, n. } 04 \text { [Accessed } 6 \\
\text { July 2021] , pp. 285-289. }\end{array}$ & $\begin{array}{l}\text { Privação do sono, sono e } \\
\text { estudantes da área da saúde }\end{array}$ & Transversal \\
\hline 12. & $\begin{array}{l}\text { Damiano, Rodolfo F. et al. The root of the problem: identifying } \\
\text { major sources of stress in Brazilian medical students and developing } \\
\text { the Medical Student Stress Factor Scale. Brazilian Journal of } \\
\text { Psychiatry [online]. 2021, v. } 43 \text {, n. } 1\end{array}$ & $\begin{array}{l}\text { Sono e estudantes da área da } \\
\text { saúde }\end{array}$ & Transversal \\
\hline 13. & $\begin{array}{l}\text { Landolt, H-P., Sousek, A. \& Holst, S. C. (2015). Effects of acute } \\
\text { and chronic sleep deprivation. In: ESRS European Sleep Medicine } \\
\text { Textbook. https://doi.org/10.5167/uzh-107182 }\end{array}$ & Privação do sono & Capítulo de livro \\
\hline 14. & $\begin{array}{l}\text { Luria, A. R. (1973). The working brain: An introduction to } \\
\text { neuropsychology. Basic books. }\end{array}$ & $\begin{array}{l}\text { Neurofisiologia e ou } \\
\text { neuropsicologia }\end{array}$ & Livro \\
\hline 15. & $\begin{array}{l}\text { Mignot E, Taheri S, Nishino S. Sleeping with the hypothalamus: } \\
\text { emerging therapeutic targes for sleep disorders. Nat Neurosci. 2002; } \\
\text { 5(suppl), }\end{array}$ & $\begin{array}{lrr}\text { Privação do } & & \text { sono, } \\
\text { neurofisiologia } & \text { e } & \text { ou } \\
\text { neuropsicologia } & & \\
\end{array}$ & Revisão \\
\hline 16. & $\begin{array}{l}\text { Pace-Schott EF, Hobson JA. The neurobiology of sleep: genetics, } \\
\text { cellular physiology and subcortical networks. Nat Rev Neurosci. } \\
\text { 2002;3(9):591-605. }\end{array}$ & $\begin{array}{lrr}\text { Privação do } & & \text { sono, } \\
\text { neurofisiologia } & \text { e } & \text { ou } \\
\text { neuropsicologia } & & \\
\end{array}$ & Revisão \\
\hline 17. & $\begin{array}{l}\text { Peever J, Fuller PM. The Biology of REM Sleep. Curr Biol. } 2017 \\
\text { Nov 20;27(22):R1237-R1248. }\end{array}$ & $\begin{array}{lrr}\begin{array}{l}\text { Privação do } \\
\text { neurofisiologia } \\
\text { neuropsicologia }\end{array} & \text { e } & \text { ou } \\
\end{array}$ & Revisão \\
\hline 18. & $\begin{array}{l}\text { Pires, G. N., Tufik, S. \& Andersen, M. L. (2012). Relationship } \\
\text { between sleep deprivation and anxiety - experimental research } \\
\text { perspective. Einstein, 10(4), 519-523. }\end{array}$ & $\begin{array}{lrr}\begin{array}{l}\text { Privação do } \\
\text { neurofisiologia }\end{array} & \text { e } & \text { ou } \\
\text { neuropsicologia } & & \end{array}$ & $\begin{array}{l}\text { Experimental } \\
\text { quantitativo }\end{array}$ \\
\hline 19. & $\begin{array}{l}\text { Ribeiro, Cairon Rodrigo Faria; Silva, Yasmin Maria Garcia Prata } \\
\text { da; Oliveira, Sandra Márcia Carvalho de. O impacto da qualidade } \\
\text { do sono na formação médica. } 12 \mathrm{n} .1 \text { (2014). Publicação Trimestral } \\
\text { - Rev Soc Bras Clin Med. - janeiro/março }\end{array}$ & $\begin{array}{l}\text { Privação do sono, sono e } \\
\text { estudantes da área da saúde }\end{array}$ & $\begin{array}{l}\text { Transversal, } \\
\text { quantitativo, } \\
\text { descritivo do tipo } \\
\text { inquérito }\end{array}$ \\
\hline 20. & $\begin{array}{l}\text { Sugahara, C., Silveira, B. F., Azevedo, A. S. F., Macena, B. B., \& } \\
\text { Aversi-Ferreira, T. A. (2021). The role of the second brain } \\
\text { functional unit II on the memory's process: A neuropsychological } \\
\text { Luria's perspective. Research, Society and Development, 10(9), } \\
\text { e27010917957. https://doi.org/10.33448/rsd-v10i9.17957 }\end{array}$ & $\begin{array}{l}\text { Neurofisiologia } \quad \text { e } \quad \text { ou } \\
\text { neuropsicologia }\end{array}$ & Revisão \\
\hline 21. & $\begin{array}{l}\text { Varga, A. W., Kang, M., Ramesh, P. V., \& Klann, E. (2014). Effects } \\
\text { of acute sleep deprivation on motor and reversal learning in mice. } \\
\text { Neurobiology of Learning and Memory, 114, 217-222. }\end{array}$ & Privação do sono & $\begin{array}{l}\text { Experimental } \\
\text { quantitativo }\end{array}$ \\
\hline
\end{tabular}


Vaz, A., L., L. et al. Fatores Associados aos Níveis de Fadiga e Sonolência Excessiva Diurna em Estudantes do Internato de um Curso de Medicina. Revista Brasileira de Educação Médica [online]. 2020, v. 44, n. 01.

Fonte: Autores.

Os dados foram plotados num gráfico em que os temas estão em intersecção (Figura 1), e discriminados em diagrama de Venn (Figura 2).

Figura 1: Porcentagem dos temas usados nessa revisão.

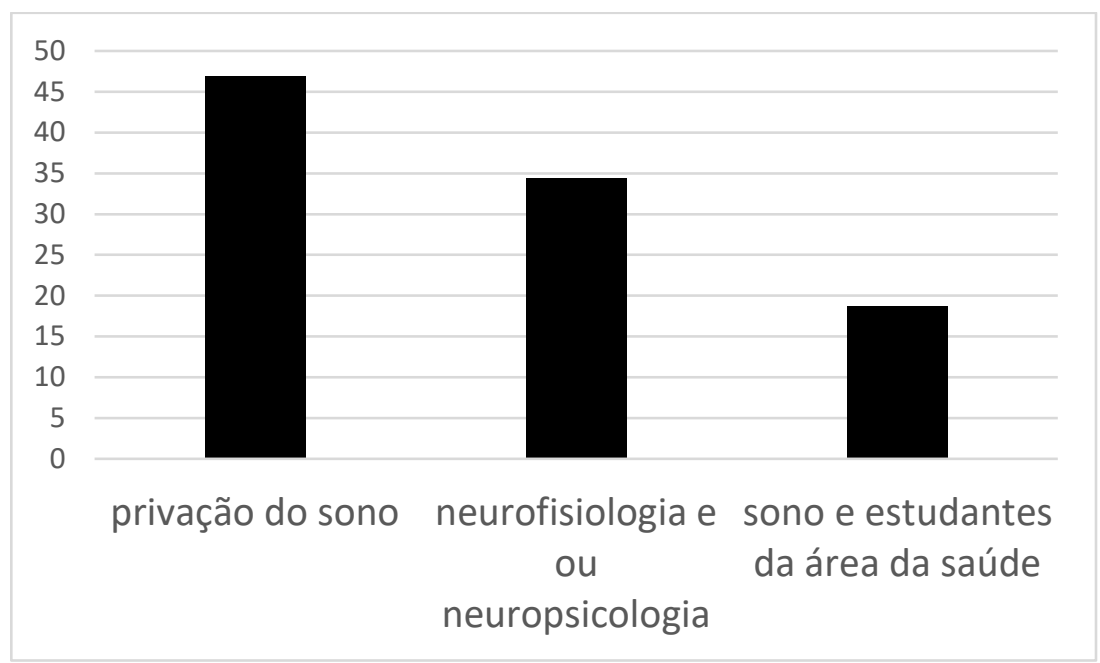

Fonte: Autores.

Figura 2: Diagrama de Venn dos temas distintos e com as intersecções dos mesmos.

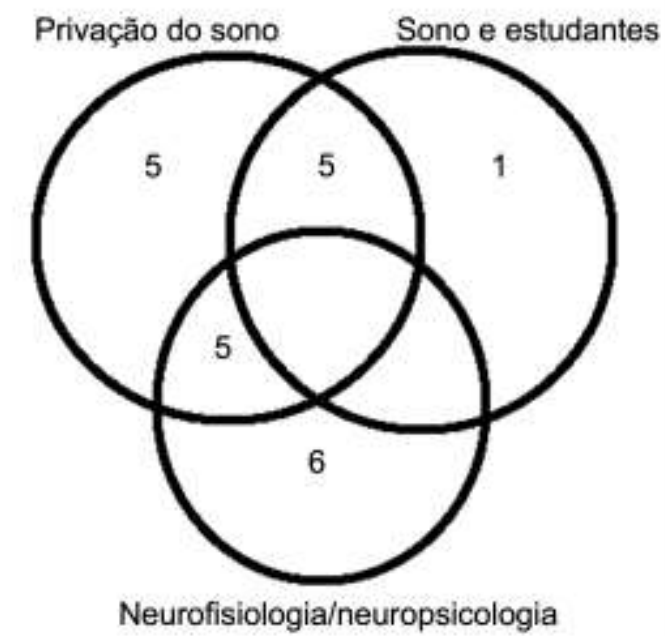

Fonte: Autores.

Os textos sobre neurofisiologia/neuropsicologia que foram usados para dar as bases de análise foram escolhidos em função da neuropsicologia fisiológica dentro do escopo das análises via lesões cerebrais que permitiram as evidências das funções cerebrais, não a neuropsicologia de testes (Sugahara et al., 2021; Cordeiro-de-Oliveira, 2021; Aversi-Ferreira et al., 2010; AversiFerreira et al., 2019; Luria, 1973). Essa base neurofuncional se mostrou necessária devido à maior parte dos artigos sobre a 
privação de sonos em estudantes terem sido feitos em estudos descritivos comportamentais sem um aprofundamento nas justificativas funcionais, sem intersecção com o tema neuropsicologia/neurofisiologia (Figura 2).

Os textos sobre privação do sono se intersectam com os estudos sobre os estudantes da área de saúde e com o tema neuropsicologia/neurofisiologia e, se referem aos problemas gerais sobre o sono como ansiedade, alterações comportamentais de efeitos sociais (Pires, Tufik \& Andersen, 2012) tem como base o aumento da sonolência com consequentes alterações metabólicas de cunho patológico (Landolt, Sousek \& Holst, 2015).

Em relação aos problemas do sono em estudantes da área de saúde, estudos foram realizados em várias universidades, todos concluindo que a privação do sono nos estudantes gera problemas de falta de atenção e diminuição da capacidade de aprender, além de observar primeiramente a quantidade de estudantes que mostraram alterações do estado de sono, logo de vigília.

De fato, mais da metade dos estudantes da Universidade de Munique mostraram distúrbios do sono em mais de $60 \%$ do grupo amostral que apresentaram redução do desempenho acadêmico sendo considerado que parte da causa é devido a um ciclo entre privação do sono e estresse com o estresse gerando menor desempenho acadêmico que causa mais estresse, logo, maior privação do sono (Ahrberg, 2012). Uma corroboração importante entre os problemas de desempenho acadêmico em relação aos problemas do sono e estresse foi realizado numa Universidade da Costa Rica (Alvarado-Fernández et al., 2012) mostrando que em estados de estresse e privação do sono em estudantes em comparação com momentos em que o estresse está menor [menos atividades e provas], logo com melhor qualidade do sono, o desempenho cognitivo do grupo estudado aumentou.

Em estudantes da Universidade Federal do Acre e Universidade de São Paulo em Botucatu foram observados problemas de origem psicossomáticos (Ribeiro, 2014), sonolência diurna e alterações circadianas (Corrêa, 2017) como consequência dos problemas do sono, e, como esperado, esses problemas se mostraram no internato médico acrescentando reclamações de fadiga observado na PUC-Goiás (Vaz, 2020).

Aspectos mais graves foram citados num estudo feito na Arábia Saudita (Al-Khani, 2019) com evidências de problemas psicossomáticos advindos da irregularidade do ciclo sono vigília como a ansiedade, depressão, estresse, e, em todos os casos citados as consequências foram devidas à má qualidade e redução do tempo de sono, comportamento também observado em estudantes que atendem em uma Unidade de Saúde da Família em Minas Gerais (Castilho, 2015).

Ficam evidentes que os estudantes apresentam as consequências da privação do sono observadas de modo geral em humanos de várias faixas etárias e gênero. Em específico, a principal reclamação dos estudantes é o estresse causado por falta de tempo de estudo, pressão infringida a si mesmo por bons resultados, pouco tempo para lazer (Damiano, 2021). As reclamações acima podem ser direcionadas para as causas de algumas patologias metabólicas devido ao aumento do tempo de vigília que desequilibra os ciclos hormonais gerais causando fome e aumento do apetite com possíveis consequências como o ganho de peso e obesidade (Alvarado-Fernández et al., 2012) e ansiedade que pode incorrer em compulsão alimentar e aumento do consumo de bebidas alcoólicas e drogas (Castilho, 2015; Al-Khani, 2019).

As bases neurofisiológicas e neuropsicológicas analisadas em detalhes oferecem explicações para os problemas já citados para a privação do sono e acrescenta dados que devem ser considerados em relação à saúde mental.

Em essência, a atividade mental exige um estado ótimo de vigília para ser dirigida a metas com a necessidade de um estado excelente de tono cortical para que uma resposta adequada seja dada a um estímulo (Luria, 1973), de fato, em um tono cortical baixo a relação excitação/inibição é diminuída.

A região cerebral responsável pelo controle do sono e da vigília envolve partes corticais e subcorticais que foram designadas por Luria (1973) de I Unidade Funcional do Cérebro Sugahara et al., 2021; Cordeiro-de-Oliveira, 2021; AversiFerreira et al., 2010; Aversi-Ferreira et al., 2019). 
O subcórtex [tálamo] e tronco cerebral [formação reticular] são essenciais para o controle do ritmo circadiano (Mignot, Taheri \& Nishino, 2002; Pace-Schott \& Hobson, 2002; Luria, 1973) mostrando uma rica conexão com o córtex cerebral recebendo e influenciando-se em duplicidade via sistema reticular ascendente e descendente (figura 3). Diferentemente de outras áreas cerebrais a formação reticular não obedece a lei tudo-ou-nada e modula gradualmente o sistema neural (Magoun \& Moruzzi, 1949).

Em termos gerais, as fibras do sistema reticular ascendente [ativador] tomam uma direção superior passando pelo tálamo, núcleo caudado, hipocampo e neocórtex que ativa o córtex e regula os estados de atividade cortical, por outro lado, as fibras reticulares descendentes [inibidor] tomam caminho reverso e modulam o estado de vigília; ambos formam um único sistema funcional autorregulador (Luria, 1973).

Figura 3: Relações corticais e subcorticais dos sistemas reticulares ascendente e descendente.

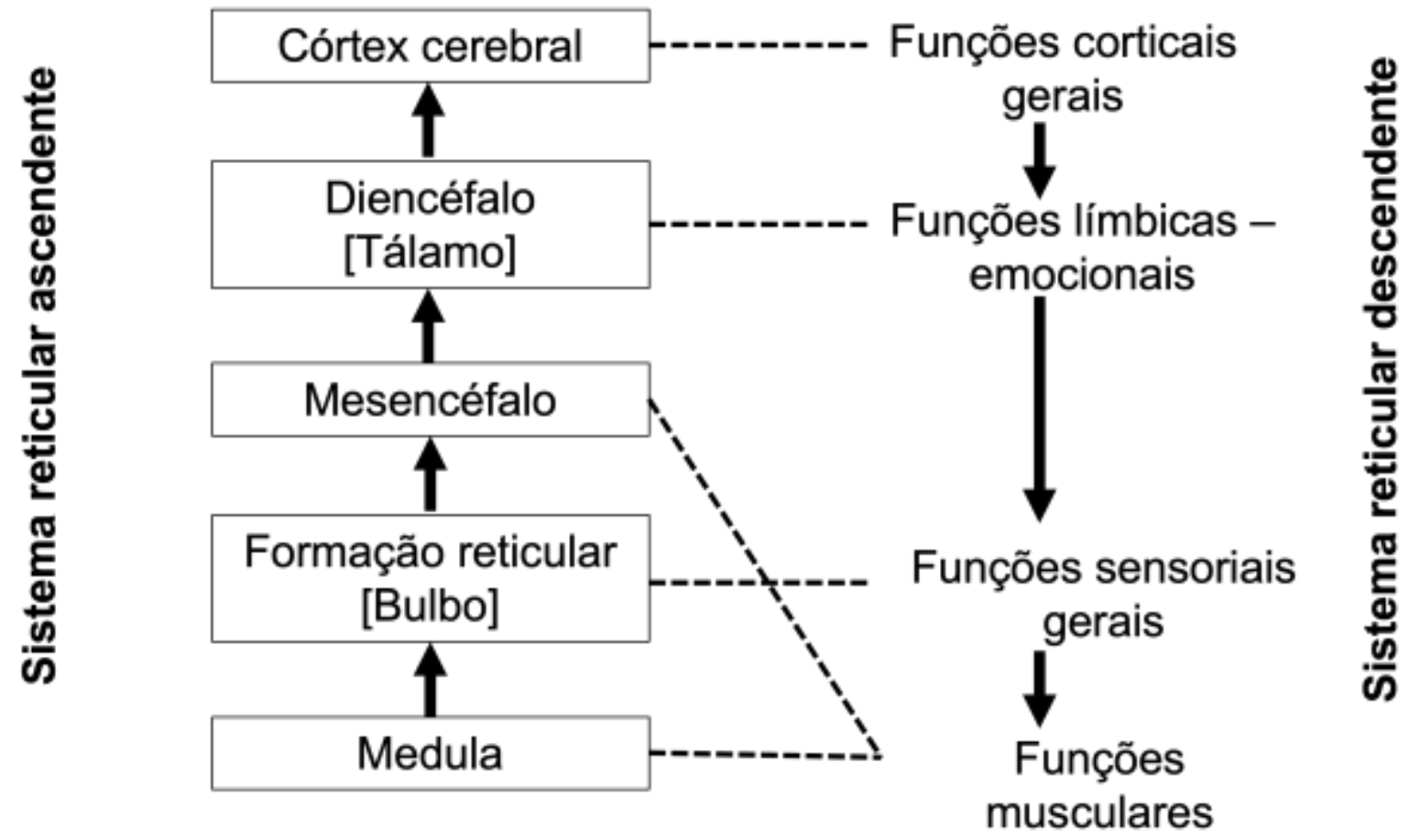

Fonte: Autores.

As bases da associação formação reticular/córtex com as estruturas intermediárias podem indicar parte dos problemas derivados do desequilíbrio do estado sono/vigília, logo, da privação do sono.

Para um ótimo estado cortical o cérebro precisa de energia que é obtida por estímulos da fome controlada pelo diencéfalo [hipotálamo] via ativação hormonal, de acordo com a atividade cerebral evocada, quanto maior a energia exigida pelo córtex cerebral, maior será o estímulo da fome, que ocorrerá mais forte em estados de vigília prolongada, como consequência da análise dos sistemas reticulares (Figura 3) evidenciando que o controle da energia para a atividade cortical é derivada de ativação/desativação metabólica.

Como a formação reticular controla o ritmo circadiano, nos momentos de sono, de menor luminosidade solar, por exemplo, ocorre uma inibição da função cortical, mas o córtex frontal, responsável pela consciência e tomadas de decisão (Cordeiro-de-Oliveira, 2021) pode optar, via estímulos conscientes, a continuação da atividade, logo estimulando a formação reticular a manter as atividades. 
Hipoteticamente, se isso ocorrer continuamente, será esperado além da fome, a compulsão por alimentos e bebidas com aumento do estresse emocional pelo fato do indivíduo estar agindo em desacordo com os parâmetros considerados normais, vendo a transformação do seu corpo, sem conseguir controlar seus estímulos devido ao já estabelecido cansaço.

Dentro do campo dos fatos, o sistema de vigília requer uma ativação/desativação do sistema límbico/hipocampal que é um dos componentes do comportamento emocional, além da parte mesial do cortex frontal. Um forte estímulo emocional age sobre o hipotálamo, em decorrência, sobre hormônios e a atividade cortical, essa última, respondendo ao estímulo de acordo com a vontade do indivíduo, que, para se controlar precisa estar em estado ótimo de sono/vigília, doutro modo, pode perder o controle emocional tendo a ansiedade como uma das consequências.

Os problemas citados de estresse, cansaço, ansiedade/depressão, perda cognitivas, dores musculares, alterações de humor/emoções podem ser explicados pela ativação/desativação mais ou menos regulada pelos sistemas reticulares ascendentes e descendentes observando as estruturas coordenadas pelo córtex frontal.

O cansaço cerebral é derivado dos estímulos excessivos de manutenção da atividade com consequente diminuição da atividade cognitiva e baixo rendimento acadêmico, alterações do humor; a privação do sono mantém o corpo por mais tempo em atividade com maior gasto de neurotransmissor para manter o tônus muscular via medula, levando ao cansaço muscular.

Concluindo em relação ao sistema de controle sono/vigília do cérebro, processos metabólicos e emocionais podem ser explicados devido à privação do sono, pelo menos em parte, no entanto, talvez mais complicado seja o problema social em relação à saúde mental.

A psicologia sócio-histórico-cultural preconiza que a mente se desenvolve não somente pelos parâmetros genéticos, mas, também pelo convívio social com a cultura sendo derivada das aquisições históricas de um povo, em termos gerais (Sugahara et al., 2021; Cordeiro-de-Oliveira, 2021; Aversi-Ferreira et al., 2019). Nesse sentido, as relações sociais são influenciadoras ou inibidoras de traços comportamentais, em consequência, um comportamento que leve à privação do sono num meio acadêmico, pode ser estimulado pelo conjunto das pessoas que sofrem do mesmo problema levando o indivíduo a seguir os padrões até mesmo para ser aceito dentro desse meio, levando a um processo comportamental em que o sofrimento pode ser visto como uma ação necessária e de coragem para a manutenção da responsabilidade derivada da profissão, e os estudantes da área de saúde estão inseridos dentro desse comportamento.

Então, as intervenções estruturais devem ser consideradas necessárias e urgentes direcionando nas causas para suprimir os efeitos, i.e., iniciar nos estudantes para evitar que os profissionais tenham patologias mais graves, visando uma melhora na qualidade de vida dos estudantes, inicialmente, consequentemente, gerando uma formação de melhor qualidade para os estudantes da área de saúde (Damiano, 2021; Correa, 2017; Vaz, 2020) que parecem sofrer mais da privação do sono.

Fica evidente que não foi possível adentrar em todas as causas da privação do sono em estudantes da área da saúde, nem das explicações cerebrais e metabólicas, não obstante os efeitos serem mais estudados nos artigos; mais trabalhos devem ser realizados para gerarem dados cuja concatenação permita criar uma estratégia bem-sucedida na prevenção da privação do sono em estudantes.

Em resposta à hipótese implícita desse trabalho, segue-se que a privação do sono altera o comportamento dos estudantes como consequências patológicas de ordem metabólica e neural/mental explicadas por uma análise de fundamentos neurofisiológicas e neuropsicológicos.

Apesar dos ainda poucos estudos sobre o isolamento social dos últimos tempos, está ocorrendo um agravo extra além das exaustivas rotinas dos estudantes devido à incidência do SARS-CoV2, que está gerando problemas na saúde mental e emocional na população mundial, logo afetando também os estudantes, o qur foi observado em um estudo desenvolvido pela Universidade do Estado da Bahia mostrando a diminuição da qualidade do sono, ansiedade, sintomas depressivos e outros transtornos psíquicos em estudantes universitários (Coelho, 2020). 


\section{Conclusão}

Através deste estudo, foi possível perceber que a qualidade do sono acomete de forma sistêmica o organismo humano, afetando diretamente o cotidiano e a qualidade de vida dos indivíduos. Os estudantes universitários das áreas de saúde, constituem um grupo especificamente vulnerável a este fenômeno, tendo em vista a carga horária elevada, situações de estresse e a própria auto-cobrança por resultados dos discentes.

A relação sono/vigilia foi o principal ponto abordado, juntamente com sua má qualidade e curso não reparador, na maioria dos casos. Entende-se a coexistência de um ciclo de piora das atividades diárias e acadêmicas em detrimento a deficiência do sono, ademais, os parâmetros físicos [metabólicos e neurais] e psicossociais são afetados.

Dentre os estudos compilados pôde-se identificar empecilhos físicos agudos, como astenia, fadiga e sonolência diurna, além dos crônicos, como: aumento de peso, obesidade e dor crônica. Outro enfoque primordial da carência na manutenção adequada do ciclo de sono/vigília, foi a constante queixa sobre o baixo rendimento escolar, com insatisfação da produtividade geral, comprovado por testes de piora da função cognitiva e da memória de curto e longo prazo. Em relação aos fatores psicológicos, é notável o aparecimento de sintomas como ansiedade, estresse e depressão frente aos agentes estressores.

Tendo em vista a trivialização e recorrente privação do sono em profissionais e estudantes da área de saúde, fazem-se necessárias e urgentes as intervenções durante todo o decorrer da formação acadêmica dos alunos, visando uma formação de melhor qualidade psicossocial. Além das alterações nas instituições acadêmicas, a esfera cultural tem poder incisivo para mudança comportamental. Ademais, ficou evidente a necessidade de mais estudos com enfoque nessa temática, uma vez que a partir da identificação dos empecilhos é possível desenvolver estratégias eficazes que melhorem a qualidade do sono e de vida dos acadêmicos.

\section{Referências}

Ackermann, S. \& Rasch, B. (2014). Differential Effects of Non-REM and REM Sleep on Memory Consolidation? Current Neurology and Neuroscience Reports, $14(2)$.

Ahrberg, K., Dresler, M., Niedermaier, S., Steiger, A., \& Genzel, L. (2012). The interaction between sleep quality and academic performance. Journal of Psychiatric Research, 46(12), 1618-1622.

Al-Khani, A. M., Sarhandi, M. I., Zaghloul, M. S., Ewid, M., \& Saquib, N. (2019). A cross-sectional survey on sleep quality, mental health, and academic performance among medical students in Saudi Arabia. BMC Research Notes, 12(665), 1-5.

Aloé, F., Azevedo, A. P. \& Hasan, R. (2005). Sleep-wake cycle mechanisms. Revista Brasileira de Psiquiatria, 27 (supl. I), 33-39.

Alvarado Fernández, V. et al., (2012). Impact of sleep deprivation on the cognitive abilities of a population of medical students. Med. Leg. Costa Rica, 29(2), 19-38

Aversi-Ferreira, T. A., Araújo, M. F. P. D., Lopes, D. B., \& Nishijo, H. (2010). History, citoarchitecture and neurophysiology of human and non human primates' parietal lobe: A review. Dementia \& neuropsychologia, 4(3), 173-180.

Aversi-Ferreira, T. A., Tamaishi-Watanabe, B. H., Magri, M. P. D. F., \& Aversi-Ferreira, R. A. (2019). Neuropsychology of the temporal lobe: Luria's and contemporary conceptions. Dementia \& Neuropsychologia, 13(3), 251-258.

Castilho, C. P., Limas, L. M. D. de, Monteiro, M. L., Silva, P. H. M. N. da, Bueno, H., \& Fari, T. A. (2015). A privação de sono nos alunos da área de saúde em atendimento nas Unidades Básicas de Saúde e suas consequências. Revista De Medicina, 94(2), 113-119.

Coelho, A. P. S. et al., (2020). Saúde mental e qualidade do sono de universitários na época da pandemia do COVID-19: experiência de um programa de assistência estudantil. Research, Society and Development, 9 (9), e943998074.

Cordeiro-de-Oliveira, K., Souza-Couto, D., Caixeta, M., Caixeta, V., \& Aversi-Ferreira, T. A. (2021). Neuropsychology of the frontal lobe and III functional brain unit: A Luria's studies and perspectives for the clinic approach. Research, Society and Development, 10(7), e48210716760. https://doi.org/10.33448/rsdv10i7.16760

Corrêa, C. C. (2017). Sleep quality in medical students: a comparison across the various phases of the medical course. Jornal Brasileiro de Pneumologia., 43, 43(4), 285-289.

Damiano, R. F. et al. (2021). The root of the problem: identifying major sources of stress in Brazilian medical students and developing the Medical Student Stress Factor Scale. Brazilian Journal of Psychiatry, 43(1), 35-42. 
Research, Society and Development, v. 10, n. 11, e326101119749, 2021

(CC BY 4.0) | ISSN 2525-3409 | DOI: http://dx.doi.org/10.33448/rsd-v10i11.19749

Landolt, H-P., Sousek, A. \& Holst, S. C. (2015). Effects of acute and chronic sleep deprivation. In: ESRS European Sleep Medicine Textbook. https://doi.org/10.5167/uzh-107182

Luria, A. R. (1973). The working brain: An introduction to neuropsychology. Basic books.

Mignot, E., Taheri. S., \& Nishino, S. (2002). Sleeping with the hypothalamus: emerging therapeutic targets for sleep disorders. Nat. Neurosci., 5(suppl):10711075 .

Pace-Schott, E. F., Hobson, J. A. (2002). The neurobiology of sleep: genetics, cellular physiology and subcortical networks. Nat Rev Neurosci., 3(9):591-605.

Peever, J., Fuller, P. M. (2017). The Biology of REM Sleep. Curr Biol., 27(22), R1237-R1248.

Pires, G. N., Tufik, S. \& Andersen, M. L. (2012). Relationship between sleep deprivation and anxiety - experimental research perspective. Einstein, 10(4), 519523.

Ribeiro, C. R. F. Silva, Silva, Y. M. G. P., \& Oliveira, S. M. C. (2014). O impacto da qualidade do sono na formação médica. Rev Soc Bras Clin Med., 12(1), 814.

Sugahara, C., Silveira, B. F., Azevedo, A. S. F., Macena, B. B., \& Aversi-Ferreira, T. A. (2021). The role of the second brain functional unit II on the memory's process: A neuropsychological Luria's perspective. Research, Society and Development, 10(9), e27010917957. https://doi.org/10.33448/rsd-v10i9.17957

Varga, A. W., Kang, M., Ramesh, P. V., \& Klann, E. (2014). Effects of acute sleep deprivation on motor and reversal learning in mice. Neurobiology of Learning and Memory, 114, 217-222.

Vaz, A. L. L. et al. (2020). Fatores Associados aos Níveis de Fadiga e Sonolência Excessiva Diurna em Estudantes do Internato de um Curso de Medicina, 44(1), $\mathrm{e} 011$. 